EPJ Web of Conferences 66, 03047 (2014)

DOI: $10.1051 /$ epjconf/ 20146603047

(C) Owned by the authors, published by EDP Sciences, 2014

\title{
Study of neutron-neutron interaction in proton pick-up reactions on ${ }^{3} \mathrm{H}$
}

\author{
E. Konobeevski ${ }^{1 a}$, V. Lebedev ${ }^{2}$, M. Mordovskoy ${ }^{1}$, A. Spasskii ${ }^{2}$, and S. Zuyev ${ }^{1}$ \\ ${ }^{1}$ Institute for Nuclear Research, Russian Academy of Sciences, 117312, Moscow, Russia \\ ${ }^{2}$ Skobel'tsyn Institute of Nuclear Physics, Moscow State University, 119991, Moscow, Russia
}

\begin{abstract}
The study of neutron-neutron interaction is performed in the reaction of proton pick-up from the triton $\mathrm{d}+{ }^{3} \mathrm{H} \rightarrow{ }^{3} \mathrm{He}+\mathrm{n}+\mathrm{n}$. The experiment will be carried out in the geometry of final state interaction measuring the relative energy of two neutrons. The simulation of the reaction showed the possibility of determining with good accuracy the energy of quasi-bound nn-singlet state, which is the main characteristic of nn-interaction. The test of the experimental technique was performed in the $\mathrm{d}+\mathrm{d} \rightarrow{ }^{2} \mathrm{He}+\mathrm{n}+\mathrm{n}$ reaction at the deuteron beam of U-120 cyclotron of Skobeltsyn Institute of Nuclear Physics.
\end{abstract}

\section{Introduction}

The analysis of data on neutron-neutron quasifree scattering allowed authors [1] to draw a conclusion about the need for a modified strengthened $n n$-interaction in the singlet ${ }^{1} S_{0}$ state:

$$
V_{\mathrm{nn}}\left({ }^{1} S_{0}\right)=\lambda \times V^{\mathrm{CDBonn}}\left({ }^{1} S_{0}\right) .
$$

This, however, leads to serious changes in the estimated nn-scattering length, accordingly to a decrease in the energy of nn-singlet state and even to an existence of bound "dineutron". H.Witala and W.Glökle [1] proposed a search for bound dineutron in $\gamma\left({ }^{3} \mathrm{H}, \mathrm{p}\right) \mathrm{nn},{ }^{3} \mathrm{H}(\mathrm{n}, \mathrm{d}) \mathrm{nn}$ and ${ }^{3} \mathrm{H}\left(\mathrm{d},{ }^{3} \mathrm{He}\right) \mathrm{nn}$ reactions. It is known that ${ }^{3} \mathrm{He}$ is principally a spatially symmetric $S$ state with its two protons mainly in opposite spin states. This component amounts for $\sim 90 \%$ of the ${ }^{3} \mathrm{He}$ wave function. Similarly, the two neutrons in ${ }^{3} \mathrm{H}$ are limited to be in a spin-singlet state. That makes the triton target (or projectile) a very suitable tool to look for an nn-singlet state in the induced breakup of ${ }^{3} \mathrm{H}$.

The idea of the experiment is the pickup of the charged particle from the triton, leaving two neutrons in the singlet state, and detects both the charged particle and one or two neutrons (or dineutron). In [2] it was noted that the breakup energy (difference between the excitation energy and the threshold of breakup into two clusters) is determined by kinetic energy of the decay particles and the difference of their emission angles. So, the excitation energy is defined as:

$$
E^{*}=E_{\mathrm{thr}}+\left[E_{1}+E_{2}-2\left(E_{1} E_{2}\right)^{1 / 2} \operatorname{Cos} \Delta \Theta\right] / 2 .
$$

Thus, measuring the energy and emitting angles of two particles ( $\mathrm{n}$ and ${ }^{3} \mathrm{He}$ ) one can reconstruct the kinematical parameters of the second neutron and determine the breakup energy for each event.

\footnotetext{
${ }^{\text {a }}$ Corresponding author: konobeev@inr.ru
} 
The analysis of breakup energy spectrum allow us to determine energy of the singlet two-neutron state and draw conclusions about the strength of the singlet component of neutron-neutron interaction.

\section{Simulation of the experiment}

A simulation of $\mathrm{d}+\mathrm{t} \rightarrow{ }^{3} \mathrm{He}^{2}{ }^{2}{ }^{*} \rightarrow{ }^{3} \mathrm{He}+\mathrm{n}+\mathrm{n}$ reaction was performed in two stages. At first stage the formation of intermediate state of a two-neutron system with excitation energy in the range $\left(E^{*}{ }_{\min }, E^{*}{ }_{\max }\right)$ was considered. Accordingly, the mass of the ${ }^{2} \mathrm{n}^{*}$ system was taken in the range of $2 m_{\mathrm{n}}+E^{*}$ min to $2 m_{\mathrm{n}}+E^{*}{ }_{\max }$. The registration angles of ${ }^{3} \mathrm{He}$ and the neutron and angular sizes of the corresponding detectors were chosen so as to provide uniform detection system efficiency over the examined range of excitation energies and appropriate energy range of secondary particles.

After that the breakup of the ${ }^{2} \mathrm{n}$ * system into two neutrons was simulated at a condition that one of neutrons is detected at the angle of emission of the two-neutron system $\Theta_{2 n}$. It is known that if the excitation energy of the system is slightly higher than the breakup threshold, the breakup particles are emitted in a relatively narrow cone of angles relative to the emission angle of the intermediate system $-\Theta_{2 \mathrm{n}}$. For example, at excitation energy of ${ }^{2} \mathrm{n}$-system $E^{*} \sim 100 \mathrm{keV}$, and energy of incident deuterons of $20 \mathrm{MeV}$ the total opening angle of neutrons is $\pm 12^{\circ}$. However, if the neutron detector is installed at an angle $\Theta_{2 \mathrm{n}}$, and its angular size is small (in our case \pm 2 ), then the second neutron also fly at close angles. In this case, the detector will register neutrons emitting only in forward or backward direction $\left(0^{\circ}\right.$ or $\left.180^{\circ}\right)$ in the ${ }^{2} \mathrm{n}$ c.m. system, then there will be two groups of neutrons of different energies, and consequently with different times-of-flight to the detector (see Fig. 1).

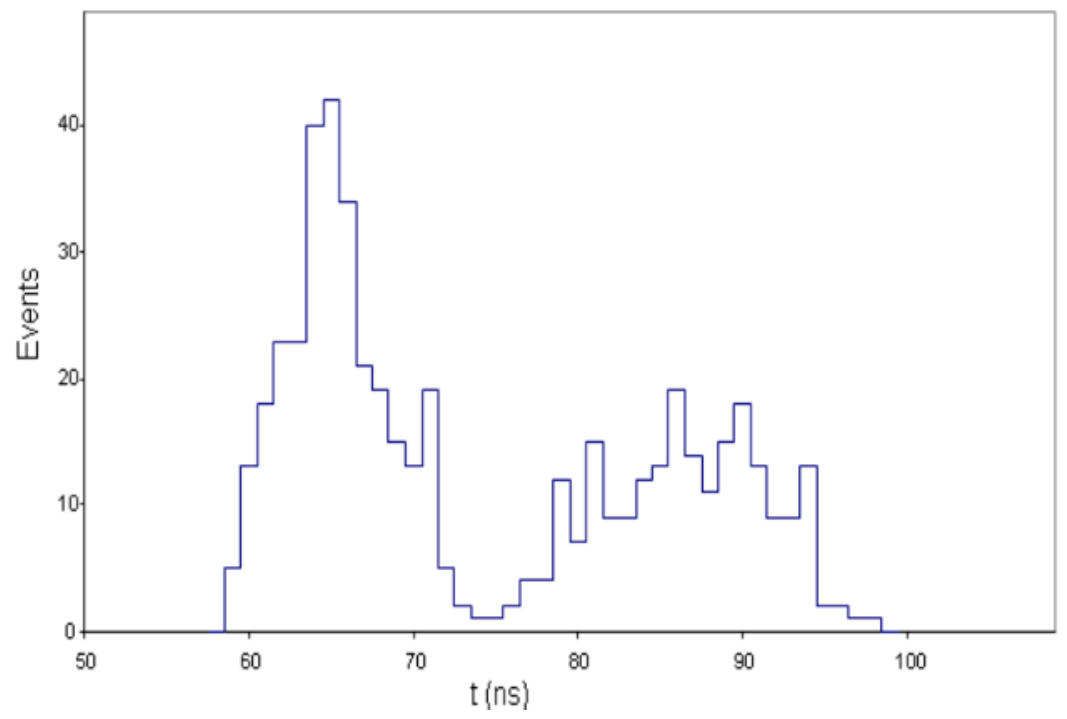

Figure 1. Time-of-flight spectrum of neutrons in ${ }^{3} \mathrm{H}\left(\mathrm{d},{ }^{3} \mathrm{He}\right) \mathrm{nn}$ reaction. $E_{\mathrm{d}}=20 \mathrm{MeV}, \Theta_{3 \mathrm{He}}=27^{\circ}, \Theta_{\mathrm{n}}=73^{\circ}$.

Such a shape of time-of-flight spectrum is characteristic for the reaction going through an intermediate state and is different from the shape of the spectrum for the usual three-body breakup (democratic breakup). In the case of registration of charged particle and only one neutron, the energy and emission angle of the second neutron will be reconstructed from the laws of energy and momentum conservation. The accuracy of reconstruction in this case will depend on the angular, energy, and time resolution of all detectors. The excitation energy for each event will be obtained using Eq. 2 with measured and reconstructed observables of both neutrons. The results of simulation show that the detection system with reasonable parameters (angular, energy and timing resolution) allows measurements over a relatively wide range of excitation energies $(20-180 \mathrm{keV})$ with a good resolution $(20-30 \mathrm{keV})$. 


\section{Testing experiment}

The test of the experimental technique was performed in the $\mathrm{d}+\mathrm{d} \rightarrow{ }^{2} \mathrm{He}+\mathrm{nn}$ reaction at $15 \mathrm{MeV}$ deuteron beam of U-120 cyclotron of Skobeltsyn Institute of Nuclear Physics.

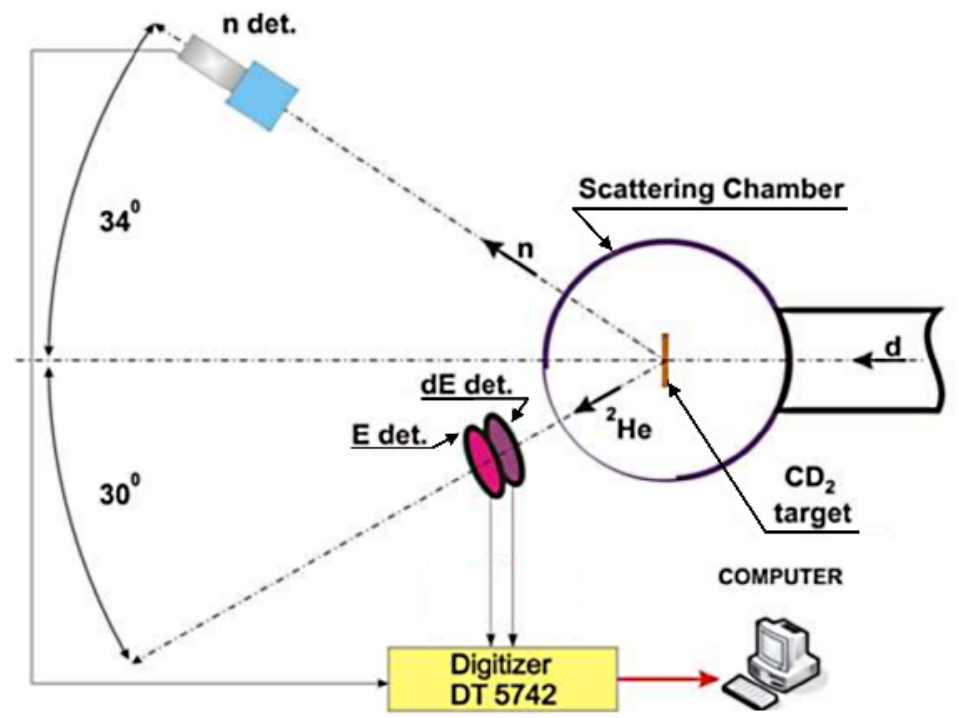

Figure 2. Layout of the experimental setup used to study the $\mathrm{d}+\mathrm{d} \rightarrow{ }^{2} \mathrm{He}+\mathrm{nn}$ reaction.

The detection system consists of two ORTEC Silicon Charged-Particle Detectors $(\Delta E$-detector with thickness of $25 \mu \mathrm{m}$ and $E$-detector with thickness of $100 \mu \mathrm{m}$ ) and a neutron detector based on liquid scintillator EJ-301 (analog of NE-213), located at angles corresponding to two-body kinematics of $d+d \rightarrow^{2} \mathrm{He}+{ }^{2} n$ reaction (see Fig. 2).

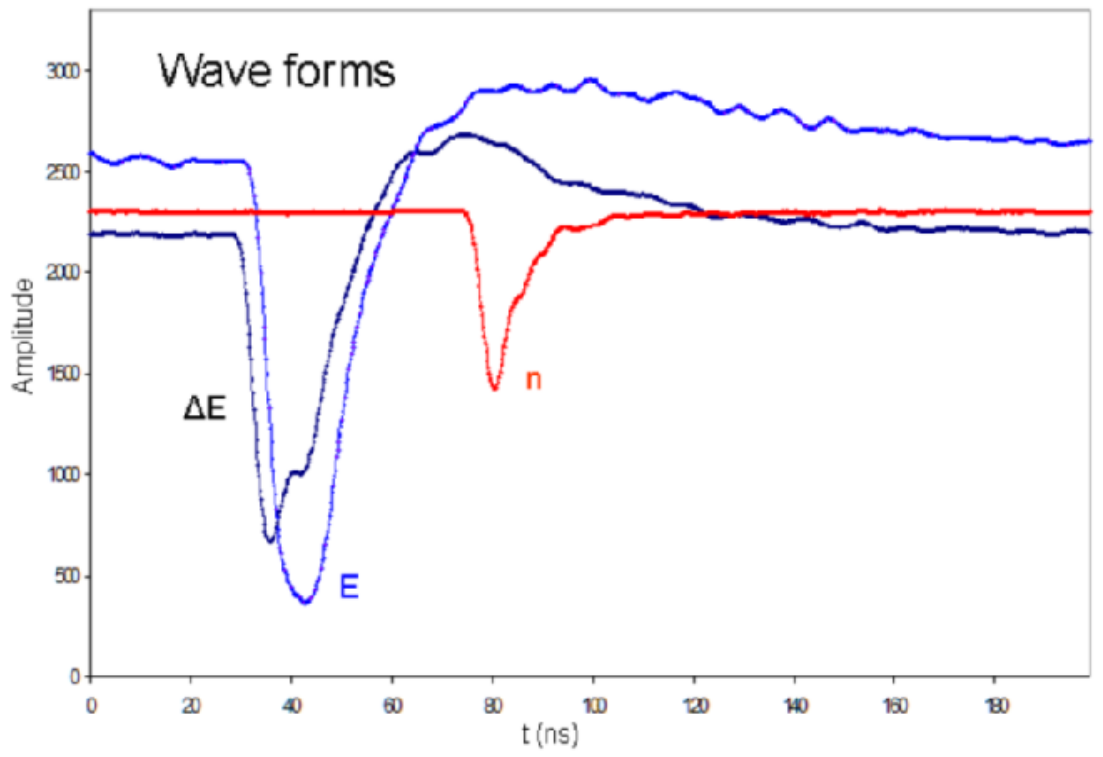

Figure 3. Wave forms of $\Delta E-, E$ - and neutron detector signals. The horizontal position of signals reflects the time of the signal formation, while the vertical amplitude reflects the energy deposited in the detector. 
As the main element of the data acquisition system we used the CAEN desktop digitizer DT5742 [3], operating as a digital oscilloscope, with the following characteristics: the number of inputs up to 16, Maximal Sampling Frequency $5 \mathrm{GS} / \mathrm{s}$ ( $0.2 \mathrm{~ns}$ between samples), amplitude resolution 12 bit. Wave forms (oscillograms) from $\Delta E$-, $E$ - and neutron-detectors (see Fig. 3) are written in the buffer memory, and on its completion are transferred to the host computer. Data processing was performed in off-line mode and consisted of determining the amplitudes and/or total areas and times of originating of signals in the detectors, digital $\Delta E-E$ and pulse shape analysis for the separation of events from different types of particles, selection of coincident events, and obtaining the final energy and time spectra for all particles. In Fig. 4 we can see very preliminary time-of-flight neutron spectrum of emitting neutron. The background events may be originated by four-body breakup $d+d \rightarrow p+p+n+n$ (democratic breakup) with registration of two protons and one neutron.

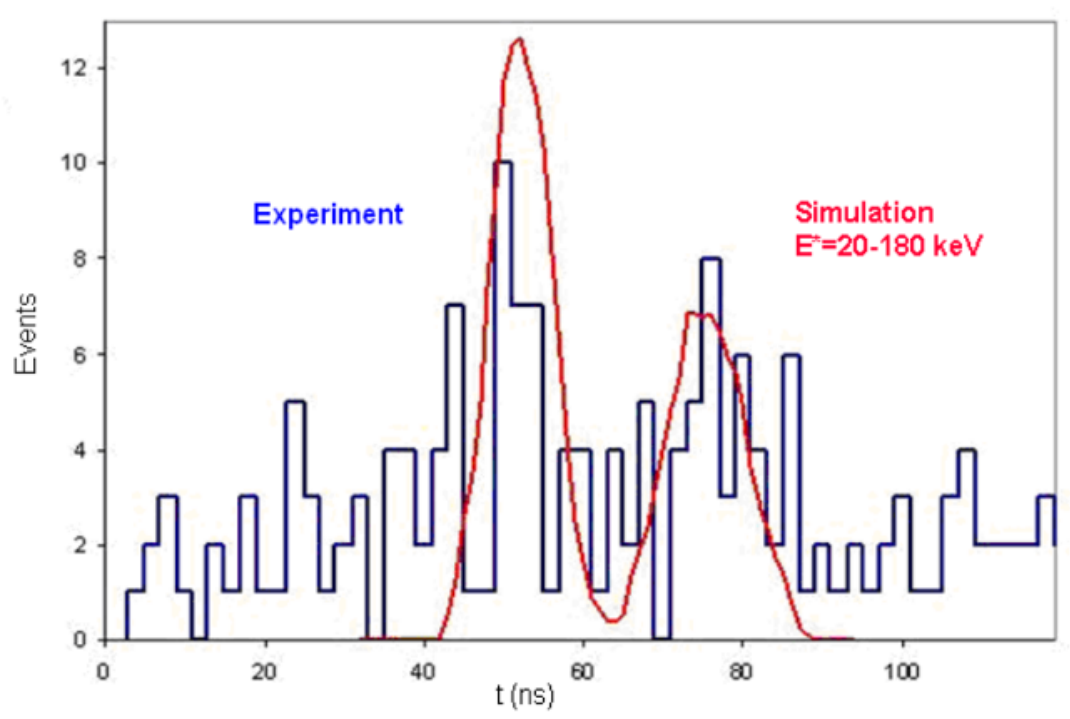

Figure 4. Simulated and experimental neutron time-of-flight spectra for ${ }^{2} \mathrm{H}\left(\mathrm{d},{ }^{2} \mathrm{He}\right) \mathrm{nn}$ reaction.

It should be noted that in the test experiment the nn-singlet state can be formed only by the chargeexchange reaction, while in ${ }^{3} \mathrm{H}$ it may initially exist due to the nn-correlations in the proton field. From the experimental point of view the test experiment is even more difficult because of the need to register two protons, and, respectively, to reconstruct the original energies of two protons from the total energies deposited in the detectors.

Data on energy and emission angles of the neutron and the charged particle in the planned experiment allow one to reconstruct the same parameters for the second neutron, and thus to obtain information on the relative energy of the two neutrons. Analysis of the dependence of the reaction yield on the relative energy will allow draw conclusions about the energy of quasibound singlet state of two neutrons and verify the theoretical basis underlying the construction of modern neutronneutron potential.

\section{References}

1. H. Witala, W. Glöckle, Phys. Rev. C83, 034004 (2011)

2. D. Robson, Nucl. Phys. A204, 523 (1974)

3. CAEN Electronic Instrumentation DT5742 digitizer, http://www.caen.it/csite/CaenProd.jsp?parent=14\&idmod=651 